\title{
Self-organizing maps could improve the classification of Spanish mutual funds
}

\author{
David Moreno ${ }^{\mathrm{a}, *}$, Paulina Marco ${ }^{\mathrm{b}}$, Ignacio Olmeda ${ }^{\mathrm{c}}$ \\ a Dpto. Economía de Empresa, Universidad Carlos III, ClMadrid 126, 28903 Getafe, Madrid, Spain \\ b Dpto. de Finanzas Empresariales, Universidad de Valencia, Avda. dels Tarongers sn, 46022 Valencia, Spain \\ ' Dpto. de Ciencias de la Computación, Universidad de Alcalá, E. U. Politécnica, Campus Universitario, \\ 28871 Alcalá de Henares, Madrid, Spain
}

\begin{abstract}
In this paper, we apply nonlinear techniques (Self Organizing Maps, $k$ nearest neighbors and the $k$ means algorithm) to evaluate the official Spanish mutual funds classification. The methodology that we propose allows us to identify which mutual funds are misclassified in the sense that they have historical performances which do not conform to the invest ment objectives established in their official category. According to this, we conclude that, on average, over $40 \%$ of mutual funds could be misclassified. Then, we propose an alternative classification, based on a double step methodology, and we find that it achieves a significantly lower rate of misclassifications. The portfolios obtained from this alternative classi fication also attain better performances in terms of return/risk and include a smaller number of assets.
\end{abstract}

Keywords: Finance; Mutual funds; Clustering; Self organizing map (SOM); Investment analysis

\section{Introduction}

In recent years, mutual funds have registered an impressive development, both in qualitative and quantitative terms, and have become one of the main investment instruments for investors around

\footnotetext{
${ }^{*}$ Corresponding author. Tel.: +34 91624 5794; fax: +34 91 6249607.

E mail address: jdmoreno@emp.uc3m.es (D. Moreno).
}

the world. For example, the Spanish mutual funds market has grown surprisingly in the last decade, thus, at the present time Spanish mutual funds markets constitute the third country in number of mutual funds in Europe and the seventh in the world. ${ }^{1}$ Given this importance, in June, 1999, an exhaustive and detailed classification of Spanish

\footnotetext{
${ }^{1}$ According to the Mutual Fund Fact Book (44th Edition, 2004) published by the Investment Company Institute.
} 
Mutual Funds was established by the CNMV ("Securities Market National Commission" in Spain) and INVERCO ("Spanish Association of Collective Investments Institutions").

From the investors' point of view, a correct classification of mutual funds is needed, because they allocate their savings in those funds that better satisfy their financial necessities. Therefore, it is extremely relevant to know which characteristics, in terms of risk-return, each mutual fund has so that the investor can select among mutual funds of different categories with the objective of diversification. Obviously, if the classification is not correctly established then investors are going to adjust their portfolios in a manner that would cause a suboptimal asset allocation.

Some authors have examined whether the categories created by grouping the mutual funds according to some of their financial attributes are in accordance with those assigned by the investment objectives (as the Spanish official classification is made). The seminal work, developed by Sharpe (1992), determined the American mutual fund styles by applying an asset class factor model. In such a model each factor represents the return on an asset class and the sensitivities (estimated parameters) are required to sum to $100 \%$. This methodology is employed to provide a natural method for constructing benchmarks, through a portfolio compounded by a mix of asset classes with the same estimated style.

Brown and Goetzmann (1997) also proposed a new classification method that tries to solve the problem of determining mutual fund styles. This method captures nonlinear patterns of return that result from active portfolio management styles. The authors find that existing classifications do a poor job of forecasting differences in future performances. Likewise, Di Bartolomeo and Witkowski (1997) perform a classification of mutual funds based on Sharpe's methodology (1992), concluding that more than one of three mutual funds is misclassified. However, the major drawback is that only returns can be employed, and no other financial characteristics from the mutual funds can be introduced in the analysis. Therefore, these methodologies cannot collect all nonlinearities that identify the real mutual fund behaviour.
More recently, Marathe and Shawky (1999) also classify American mutual funds employing a cluster methodology. They find that $43 \%$ of the mutual funds do not belong to their stated categories and that in many instances self-declared categories of mutual funds are indistinguishable from one another when their classification is based on financial characteristics. Also, Kim et al. (2000) classify American mutual funds by employing a discriminant analysis and find that the stated objectives of more than half the mutual funds differ from attributes-based objectives, and over one third of the funds are severely misclassified.

According to all these authors, the current system of classifying mutual funds which is based on their stated objectives has significant room for improvement. This provides impetus to research on better and more careful fund classification techniques to ensure that the funds do indeed stay true to their stated objectives. In accordance with the above idea, in this paper we apply a very powerful nonlinear methodology to classify mutual funds, which takes into account all nonlinear patterns and relations between all the funds' attributes. This methodology allows us to identify the misclassified mutual funds, and also, to reclassify them. This alternative classification seems to achieve better diversification performances with a lower number of assets, which permits, in general, an increase in the final utility of particular investors.

In our view, the results presented here have relevant implications. For example, knowing the correct group in which a mutual fund should be classified is extremely relevant for performance persistence studies (e.g. Grinblatt and Titman, 1992; Brown and Goetzmann, 1995 or Carhart, 1997; among others). They analyze whether those mutual funds, which were winners during the previous period, continue to be winners in the next one. Obviously, in this type of study, it is critical to know the group in which each mutual fund is really allocated according to its financial characteristics instead of the declaration made by fund managers when the mutual fund was born. In addition, grouping mutual funds correctly may have several implications for ex post and ex ante benchmarks and the construction and evaluation 
Table 1

Requisites for the official classification (CNMV)

\begin{tabular}{|c|c|c|c|c|c|c|}
\hline $\begin{array}{l}\text { Denomination } \\
\text { in the paper }\end{array}$ & Category & $\begin{array}{l}\text { Percentage invested in } \\
\text { treasury bills and } \\
\text { bonds }\end{array}$ & $\begin{array}{l}\text { Average } \\
\text { duration } \\
\text { of bonds }\end{array}$ & $\begin{array}{l}\text { Percentage } \\
\text { invested } \\
\text { in stocks }\end{array}$ & $\begin{array}{l}\text { Proportion } \\
\text { invested } \\
\text { in currencies } \\
\text { different } \\
\text { from euro }\end{array}$ & $\begin{array}{l}\text { Proportion } \\
\text { of assets } \\
\text { traded in } \\
\text { OTC markets }\end{array}$ \\
\hline I & FIAMM & $100 \%$ & $<1.5$ years & & $<5 \%$ & $<5 \%$ \\
\hline II & Global Mutual Funds & \multicolumn{5}{|c|}{$\begin{array}{l}\text { They do not have any explicit restriction from the CNMV. They usually are } \\
\text { characterized by a high level of risk exposition }\end{array}$} \\
\hline IV & FIM R.F. Insured & \multicolumn{5}{|c|}{ They ensure a profit at the end of some time period. } \\
\hline V & FIM R.F.M. & $>70 \%$ & & $<30 \%$ & $<5 \%$ & $<10 \%$ \\
\hline VI & FIM R.F.M. International & $>70 \%$ & & $<30 \%$ & $>5 \%$ & $<10 \%$ \\
\hline VII & FIM R.V. Euro & $<25 \%$ & & $>75 \%$ & $<30 \%$ & $<10 \%$ \\
\hline
\end{tabular}

VIII FIM R.V. Insured

IX

$\mathrm{X}$

$\mathrm{XI}$

XII

XIII

XIV
FIM R.V. National

FIM R.V. International ${ }^{\mathrm{a}}$

FIM R.V.M.

FIM R.V.M. International

FIM R.F. International

FIM R.F. long term

They ensure a profit at the end of some time period. This return can be fix or variable, which is associated to some index

\begin{tabular}{|c|c|c|c|c|}
\hline$<25 \%$ & & $>75 \%$ & $<30 \%$ & $<10 \%$ \\
\hline$<25 \%$ & & $>75 \%$ & $>30 \%$ & $<10 \%$ \\
\hline $2570 \%$ & & $3075 \%$ & $<30 \%$ & $<10 \%$ \\
\hline $2570 \%$ & & $3075 \%$ & $>30 \%$ & $<10 \%$ \\
\hline $100 \%$ & & $0 \%$ & $>5 \%$ & $<10 \%$ \\
\hline $100 \%$ & $>2$ years & $0 \%$ & $<5 \%$ & $<10 \%$ \\
\hline
\end{tabular}

This table shows the main characteristics and requirements of the different categories of Spanish mutual funds. A mutual fund is classified in one of these categories or groups according to the type of assets and proportions hold by the mutual fund. In the first column a shorter name is established to be able to recognize each mutual fund category in the paper.

${ }^{a}$ In this category we include mutual funds which invest internationally in emerging markets, Japanese market or American market.

of performance measures (see Grinblatt and Titman, 1994).

The remainder of this paper is organized as follows. In the next section we describe the dataset used. In Section 3 we briefly describe the models employed, $k$-nearest neighbors (KNN), Self-Organizing Maps (SOM) and the $k$-means clustering algorithm. In Section 4 we detail methodology and main results. Section 5 concludes the paper.

\section{Mutual fund database}

Our database consists of 1592 mutual funds from the Spanish market, which contains weekly data returns (net of expenses and trading cost) from 1st January 2000 until January 2003, representing approximately seventy per cent of the whole of the Spanish mutual funds universe
(2333 funds). ${ }^{2}$ Mutual funds are classified into 14 different legal categories in Spain. These categories, as we mentioned above, were established by the CNMV and INVERCO in June, 1999, and constitute the actual and official classification. ${ }^{3}$ In Table 1, a survey of these 14 categories and

\footnotetext{
${ }^{2}$ An important characteristic of this data is that it is almost free of survivorship bias (see Brown et al. (1992) for more details), given that for the time period considered only nineteen funds (among all mutual funds categories) were dropped out of Spanish mutual funds market. Also note that the period considered corresponds to a "bear market", so that our conclusions should be interpreted with some caution. We appreciate one of the anonymous referees for pointing out this fact.

${ }^{3}$ Given that the actual classification in Spain started in June 1999, we must constraint the beginning of our data to that date.
} 
Table 2

Main statistics of mutual funds categories

\begin{tabular}{|c|c|c|c|c|c|c|c|c|c|c|c|}
\hline & Return & Return3 & Risk & Risk3 & Skewness & Kurtosis & $\begin{array}{l}\text { Max. } \\
\text { losses }\end{array}$ & $\begin{array}{l}\text { Max. } \\
\text { returns }\end{array}$ & $\begin{array}{l}\text { Number } \\
\text { of funds }\end{array}$ & Representativeness & $\begin{array}{l}\text { Test } \\
\text { normality }\end{array}$ \\
\hline I & 2.29 & 8.85 & 0.13 & 0.18 & 0.20 & 6.53 & 0.01 & 0.10 & 124 & 80.52 & 78.23 \\
\hline II & 11.67 & 21.30 & 11.64 & 12.82 & 0.32 & 4.55 & 3.61 & 2.78 & 57 & 58.76 & 94.74 \\
\hline III & 2.07 & 8.69 & 1.00 & 1.01 & 0.18 & 5.96 & 0.20 & 0.29 & 129 & 70.88 & 88.37 \\
\hline IV & 3.60 & 11.79 & 1.23 & 1.42 & 0.09 & 6.53 & 0.29 & 0.42 & 150 & 70.42 & 98.00 \\
\hline V & 3.39 & 4.17 & 5.20 & 4.90 & 0.25 & 4.25 & 1.33 & 1.11 & 121 & 73.78 & 84.30 \\
\hline VI & 3.62 & 3.44 & 4.69 & 5.00 & 0.21 & 4.61 & 1.32 & 1.17 & 53 & 64.63 & 77.36 \\
\hline VII & 24.17 & 39.25 & 24.33 & 20.16 & 0.21 & 4.23 & 5.57 & 4.43 & 71 & 76.34 & 94.37 \\
\hline VIII & 0.43 & 2.76 & 3.36 & 5.11 & 0.59 & 8.15 & 1.45 & 1.16 & 274 & 74.66 & 97.08 \\
\hline IX & 7.59 & 15.63 & 11.95 & 11.53 & 0.34 & 6.33 & 3.27 & 2.46 & 36 & 39.13 & 94.44 \\
\hline$X$ & 27.57 & 47.36 & 25.64 & 24.54 & 0.13 & 3.78 & 6.73 & 5.39 & 204 & 52.99 & 74.51 \\
\hline XI & 16.41 & 28.43 & 16.45 & 15.08 & 0.25 & 3.85 & 4.15 & 3.31 & 100 & 52.91 & 85.00 \\
\hline XII & 15.95 & 27.90 & 14.27 & 13.14 & 0.23 & 3.79 & 3.66 & 2.86 & 76 & 79.17 & 73.68 \\
\hline XIII & 10.83 & 14.29 & 9.21 & 9.64 & 0.14 & 3.75 & 2.58 & 2.17 & 48 & 84.21 & 60.42 \\
\hline XIV & 3.11 & 12.30 & 2.14 & 2.31 & 0.16 & 4.39 & 0.52 & 0.63 & 149 & 91.98 & 91.28 \\
\hline
\end{tabular}

The table shows the major characteristics of mutual funds in the database: the mean return for the last year (return), the accumulated return for the whole time span (return3), the risk (measured as the annualized standard deviation) for the last year (risk) and for the whole time span (risk3), the skewness (it is the third central moment divided by the cube of the standard deviation), the kurtosis (measured as the fourth central moment divided by the square of the variance of the data), the weekly minimum return (Max. Losses) and the weekly maximum return (Max. Return) during all the sample. Also we present the number of mutual funds in each category (Number of Funds) and the percentage that our data represents of the total number of mutual funds in Spanish market (Represen tativeness). Finally, the last column (Test Normality) represents the percentage of funds for which the null hypothesis of normality of a Jarque Bera test is rejected at a ten percent level of significance.

their main characteristics is shown. ${ }^{4}$ Mutual funds are classified according to the investment objectives declared by the mutual fund manager when the fund is born. Thus, the mutual fund is classified according to the type of assets and there proportion (according to Table 1) in which the mutual fund manager declares that he will invest.

A complete description (economical and statistical) of the database is shown in Table 2. In columns, we show for each category: the mean return for the last year (return), the mean return for the whole time span (return3), the risk, measured as the annualized standard deviation for the last year (risk) and for the whole time span (risk3), the skewness, the kurtosis, the weekly minimum and maximum return for all the sample, the number of mutual funds in each category, the percentage that our data represents of the total number of funds in the Spanish

\footnotetext{
${ }^{4}$ As it can be seen, some of the official categories do not have any special and defined requirements, as Global Mutual Funds. However, we decided to include these mutual funds in order to really know what their behavior is in a financial framework, given its relevance for investors.
}

market. We also show the percentage of funds for which the null hypothesis of normality, from a Jarque Bera test, is rejected at a ten percent significance level. We can see that on average the equity funds show a negative return in Table 2, this happens because the time period considered was a bear market for stocks.

For the rest of the paper, we consider a total of ten fund attributes, which we group in four different sets. These attributes are: (1) average return, (2) standard deviation, (3) skewness, (4) kurtosis, (5) the five percent of maximum losses, ${ }^{5}(6)$ the five percent of maximum returns, (7) the Reward-toSemivariability ratio, ${ }^{6}(8)$ the Beta (Sharpe, 1964)

\footnotetext{
${ }^{5}$ We use these attributes at $5 \%$ inspired by the Value at Risk methodology which is commonly used in finance to evaluate the level of risk of a portfolio.

${ }^{6}$ We consider this ratio instead of the Sharpe's Ratio because of the non normality found in the mutual funds returns. A common mistake in the literature is to consider Sharpe's Ratio when the returns distribution is non normal. As we can see from Table 2 more than $85 \%$ of funds reject the null hypothesis of normality so that Sharpe's ratio would induce an important bias.
} 
against the IBEX-35, (9) the Beta against a Notional Bond of 10 years duration and (10) the correlation of each fund with an equally weighted benchmark obtained from each of the 14 official categories. Notice that all of the above attributes were computed for both the last year and the whole time span (3 years). ${ }^{7}$

\section{Clustering techniques employed}

In this paper we use two different clustering techniques: the $k$-nearest neighbors algorithm, and a two-level approach, where the data is first clustered using the Self Organizing Map and then the results are refined using the $k$-means algorithm. Given that the results of clustering algorithms may be highly dependent on the scale of the data set used, in this paper we decide to normalize all the variables subtracting the mean and dividing by the standard deviation.

\subsection{The k-nearest neighbors (KNN) algorithm}

The $k$-nearest neighbor (KNN) algorithm is a particularly simple yet powerful method, independently proposed in machine learning and in the non-parametric literature. ${ }^{8}$ This algorithm is "memory based" so that a particular model is not properly fitted. Given a particular input $x_{0}$ we find the $k$ training points $x(r), r \quad 1, \ldots, k$ that are closest, according to a particular distance, to $x_{0}$. In this paper we employ the euclidean distance $d_{(i)}=\left\|x_{0}-x_{(i)}\right\|$

which is a common choice found in the literature. The input $x_{0}$ is classified using a majority vote among the $k$ neighbors and ties are broken at random. In order to avoid problems due to the different units of the features, the data is first

\footnotetext{
${ }^{7}$ Contrary to some other studies (e.g. Kim et al., 2000) we do not take the total asset value of mutual funds as a relevant variable for clustering since we consider that it does not reflect any specific behavior of funds in a financial framework given that the liquidity is always guaranteed in the Spanish mutual funds market.

${ }^{8}$ See Dasarathy (1991) and Aha (1990) for a survey.
}

standardized so that all the features have mean zero and unit variance.

\subsection{The self-organizing map}

The Self-Organizing Map (SOM), also called Kohonen map, is a popular feed forward Artificial Neural Network (ANN) based on unsupervised learning which has properties of both vector quantization (Gray, 1984) and vector projection algorithms (Kaski, 1997). The SOM, through a process called self-organization, configures the output units into a topological representation of the original data, positioning the prototype vectors on a regular low-dimensional grid in an ordered fashion, making the SOM a powerful visualization tool. $^{9}$

The number of neurons can vary from a few dozen up to several thousand. They are connected to adjacent neurons by a neighborhood relation, dictating the topology of the map. Each neuron $i$ has an associated $d$-dimensional prototype or codebook vector, $m_{i} \quad\left[m_{i 1}, m_{i 2}, \ldots, m_{i d}\right]$. The dimension $d$ is the same as the input vector's dimension.

The SOM is trained iteratively. In each training step, one sample vector $x$ from the input data set is chosen randomly and the similarity between it and all prototypes of the map are calculated using a certain distance measure, typically Euclidian distance. The unit whose incoming connection weights have the greater similarity with the input pattern $\boldsymbol{x}$ is called the Best-Matching Unit (BMU), denoted as $c$ :

$\left\|x-m_{c}\right\|=\min _{i}\left\{\left\|x-m_{i}\right\|\right\}$,

where $\|\cdot\|$ is the distance measure.

After finding the BMU, the prototype vectors (or connection weights) of the SOM are adjusted. SOM creates a topological mapping by updating not only the BMU's weights, which are adjusted (i.e. moved in the direction of the input pattern by a factor determined by the learning rate), ${ }^{10}$ but also adjusting the weights of the adjacent

\footnotetext{
${ }^{9}$ For a more complete description about the SOM, see Kohonen (1997) or Deboeck and Kohonen (2000).

${ }^{10}$ This is the basic nature of competitive neural networks.
} 
output units in close proximity to the neighbourhood of the winner. So not only does the BMU get updated, but the whole neighbourhood of output neurons gets moved closer to the input pattern.

The SOM update rule for the weight vector of neuron $i$ is:

$m_{i}(t+1)=m_{i}(t)+\alpha(t) h_{c i}(r(t))\left[x(t)-m_{i}(t)\right]$,

where $t$ denotes time, $\alpha(t)$ is the learning rate, that is a monotonically decreasing function of time between $(0,1)$, and $h_{c i}(r(t))$ the neighbourhood kernel around the BMU $c$, with neighbourhood radius $r(t)$, which typically decreasess with time. ${ }^{11}$

\subsection{The k-means clustering algorithm}

The $k$-means constitute one the most popular methods for multidimensional data clustering. It is an iterative relocation algorithm, which works in a partitioning way. This algorithm minimizes the sum of the within group distances between all points and the cluster center (centroid) to get the best classification of the data.

Following Tou and González (1974), the procedure in which this algorithm works can be defined as follows:

(i) $K$ initial centers $z_{1}(1), z_{2}(1), \ldots, z_{k}(1)$ are chosen. They may or may not be the centroids.

(ii) All the data points are assigned to clusters. At the $k$ th iterative step, each point of the whole sample is assigned to one of the $K$ clusters according to the following relation:

$x \in C_{j}(k)$ if $\left\|x-z_{j}(k)\right\|<\left\|x-z_{i}(k)\right\|$

for all $i \quad 1,2, \ldots, K ; i \neq j$; where $C_{j}(k)$ denotes the set of samples whose centroid is $z_{j}(k)$.

(iii) Next, the new centroids are computed $\left[z_{j}(k+1), j \quad 1,2, \ldots, K\right]$ such that the sum of the squared distances from all points in

11 The neighbourhood kernel is a non increasing function of time and of the distance of neuron $i$ from the winner unit $c$ :

$h_{c i}(r(t)) \quad \exp \left(\frac{\left\|r_{c} r_{i}\right\|^{2}}{2 \sigma^{2}(t)}\right)$,

where $r_{c}$ and $r_{i}$ are positions of units $c$ and $i$ on the SOM grid.
$C_{j}(k)$ to the new cluster center is minimized. Knowing that the data mean is the measure which minimizes this distance, then, the new cluster center is:

$z_{j}(k+1)=\frac{1}{N_{j}}\left(\sum_{x \in C_{j}} x\right) j=1,2, \ldots, K$,

where $N_{j}$ is the number of data in the new cluster $C_{j}(k+1)$.

(iv) If no further improvement is achieved, i.e., $z_{j}(k+1) \quad z_{j}(k)$ for all $j$, the algorithm has converged and the procedure is finished. Otherwise, all steps from number ii must be realized again.

As can be noted, the configuration of the final clustering will depend on the initial centroids and on the number of $K$. To solve this problem, we employ a common measure, the Davies Bouldin Index (1979). This index has the objective of minimizing the within-cluster distance and maximizing the between-cluster separation. ${ }^{12}$ According to this index, the best clustering should minimize the next expression:

$\frac{1}{C} \sum_{k}^{C} \max _{k / p}\left\{\frac{S_{c}\left(Q_{k}\right)+S_{c}\left(Q_{p}\right)}{d_{c e}\left(Q_{k}, Q_{p}\right)}\right\}$,

where $S_{c}$ is the within-cluster distance, $d_{c e}$ is the between-clusters distance and $C$ is the number of clusters.

\section{Methodology and results}

The methodology and results, in this paper, can be divided into five different steps.

\subsection{Evaluation of the stated categories (Step 1)}

Firstly, we evaluate the 14 official mutual funds categories. We employ the KNN algorithm and a

\footnotetext{
12 Although, several other measures have been proposed to achieve the same goal (see Bezdek and Pal, 1998) we decided to employ this because it is followed by Vesanto and Alhoniemi (2000) as a complementary tool to Self Organizing Maps (SOMs).
} 
sampling technique closely related to jackknife $e^{13}$ to evaluate the percentage of misclassified mutual funds. The methodology of this first step is as follows:

(i) We train the KNN algorithm for the whole sample except for one fund (mutual fund $i$ ) using a set of attributes as the independent variables and using the official classification of the fund as the dependent one.

(ii) Next, we present mutual fund $i$ to the KNN which classifies it into some of the 14 official groups. Then, we compute if it is correctly classified or not and, if it is misclassified, we find in which category it has been (wrongly) placed.

(iii) After that, we repeat the steps (i) and (ii) for another fund (mutual fund $j \neq i$ ), until all mutual funds have been classified.

As can be seen, it is a very intensive computational process, because we have to train the KNN algorithm a number of times equal to the number of mutual funds in our database (1592 times). In addition, it must be noted that we perform this experiment for different sets of variables with the aim of demonstrating that the results are robust and are not different depending on the variables used. In particular, we consider four different groups of attributes: ${ }^{14}$

(a) Group I: The return and risk for the last year and for the entire sample (four attributes).

(b) Group II: The attributes of Group I, the Skewness and the Kurtosis for the last year and the entire sample (eight attributes).

(c) Group III: Those in Group I and the correlation of each mutual fund with an equally weighted benchmark from each official category, computed both for the last year and for the entire sample (32 attributes).

\footnotetext{
${ }^{13}$ The difference between jacknife and the proposed procedure is that jacknife samples with replacement from an, in princi ple infinite universe while we sample without replacement from a finite one. For more details see Efron and Tibshirani (1993).

${ }^{14}$ It must be pointed out that these groups are maintained during each one of the first four steps in which we perform our study.
}

(d) Group IV: The attributes of Group I, the five percent of maximum losses, the five percent of highest gains, the reward-to-semivariability ratio, the beta against the IBEX-35 index and against a Notional Bond index, each of them computed both for the last 3 years and for the last one (14 attributes).

We show the results of these experiments in Tables $36{ }^{15}$ As a general result, we observe that the categories I, VIII and X achieve the highest rate of funds correctly classified. It is interesting that Group X (FIM RV International) is a very homogeneous category, in spite of including mutual funds which invest internationally in emerging markets, American market, Japanese market, etc. (see Table 1). Similarly, even though the category VIII (FIM R.V. Insured) does not have very restrictive requirements, the majority of mutual funds included there seem to have very homogeneous behavior.

Also, from Tables 36 we find that categories II and IX (Global Mutual Funds, and FIM R.V. National, respectively) are the groups with the highest rate of misclassifications (This results are more easily observed in Fig. 1, which shows the rate of misclassified mutual funds from each official category and using the four different group of attributes consider in the study). On average, they achieve only a $7.89 \%$ and $1.39 \%$ respectively, of mutual funds correctly classified. According to Table 1, it seems logical that the category II achieves those poor results because the official classification really does not impose any requirements in this category. However, category IX has strict requirements (i.e. the percentage of equity must be higher than $75 \%$ of the total portfolio value) but their mutual funds are systematically misclassified.

If we make a more detailed analysis, we can observe how the majority of funds from category IX

\footnotetext{
${ }^{15}$ We compute the experiment for $1,5,10,15$ and 25 neighbors, using a majority vote rule and a random choice in case of ties. We only presents the results from a specification (it is, when five neighbors are employed in the $\mathrm{KNN}$ algorithm) because the rest of results are all very similar, but they are available upon request.
} 
Table 3

Percentage of mutual funds correctly classified according to KNN technique

\begin{tabular}{|c|c|c|c|c|c|c|c|c|c|c|c|c|c|c|}
\hline & I & II & III & IV & $\mathrm{V}$ & VI & VII & VIII & IX & $\mathrm{X}$ & XI & XII & XIII & XIV \\
\hline I & 87.90 & 0.00 & 10.48 & 1.61 & 0.00 & 0.00 & 0.00 & 0.00 & 0.00 & 0.00 & 0.00 & 0.00 & 0.00 & 0.00 \\
\hline II & 7.02 & 3.51 & 0.00 & 1.75 & 5.26 & 1.75 & 1.75 & 17.54 & 1.75 & 19.30 & 14.04 & 15.79 & 5.26 & 5.26 \\
\hline III & 17.05 & 0.00 & 53.49 & 11.63 & 1.55 & 0.00 & 0.00 & 0.78 & 0.78 & 0.78 & 0.78 & 0.00 & 0.00 & 13.18 \\
\hline IV & 2.00 & 0.00 & 10.00 & 59.33 & 2.67 & 0.00 & 0.00 & 6.00 & 0.00 & 0.00 & 0.00 & 0.00 & 0.00 & 20.00 \\
\hline V & 0.00 & 1.65 & 4.13 & 4.96 & 43.80 & 8.26 & 2.48 & 20.66 & 0.00 & 0.00 & 4.96 & 0.83 & 3.31 & 4.96 \\
\hline VI & 0.00 & 1.89 & 0.00 & 0.00 & 45.28 & 16.98 & 3.77 & 16.98 & 0.00 & 0.00 & 3.77 & 0.00 & 5.66 & 5.66 \\
\hline VII & 0.00 & 0.00 & 1.41 & 0.00 & 8.45 & 2.82 & 45.07 & 4.23 & 0.00 & 26.76 & 5.63 & 2.82 & 2.82 & 0.00 \\
\hline VIII & 0.00 & 1.09 & 0.73 & 1.46 & 7.30 & 2.19 & 2.19 & 77.74 & 1.09 & 1.82 & 0.00 & 1.09 & 2.55 & 0.73 \\
\hline IX & 0.00 & 2.78 & 2.78 & 0.00 & 2.78 & 2.78 & 0.00 & 52.78 & 2.78 & 27.78 & 2.78 & 2.78 & 0.00 & 0.00 \\
\hline $\mathrm{X}$ & 0.00 & 2.45 & 0.00 & 0.00 & 0.00 & 0.00 & 5.39 & 1.96 & 1.47 & 80.88 & 2.45 & 3.43 & 1.47 & 0.49 \\
\hline XI & 0.00 & 2.00 & 0.00 & 0.00 & 9.00 & 1.00 & 2.00 & 3.00 & 0.00 & 19.00 & 39.00 & 20.00 & 5.00 & 0.00 \\
\hline XII & 0.00 & 3.95 & 0.00 & 0.00 & 6.58 & 2.63 & 0.00 & 3.95 & 0.00 & 10.53 & 34.21 & 35.53 & 2.63 & 0.00 \\
\hline XIII & 0.00 & 6.25 & 2.08 & 0.00 & 18.75 & 4.17 & 0.00 & 2.08 & 0.00 & 8.33 & 12.50 & 4.17 & 25.00 & 16.67 \\
\hline XIV & 0.00 & 0.00 & 14.09 & 18.79 & 3.36 & 0.67 & 0.00 & 4.03 & 0.00 & 0.00 & 0.00 & 0.00 & 4.70 & 54.36 \\
\hline
\end{tabular}

Employing five neighbours and the attributes of Group I.

This table shows the correspondence between the official categories and the attributed based classification. Each cell $(i, j)$ shows the percentage of the mutual funds from the legal category $i$ (rows) that are classified (from a $k$ nearest neighbours technique) in the category $j$ (columns). Thus, the diagonal (in boldface) represents the percentage of mutual funds which are correctly classified in the official classification.

are classified in Groups VIII and X (FIM R.V. Insured and FIM R.V. International, respectively). ${ }^{16}$ In addition, it must be noted that this high rate of misclassifications could be due to the small number of funds in this category (in our database, they are represented only by 36 funds, that is, $39 \%$ of the total number of funds in this category of the Spanish market).

In Fig. 1 we can observe more clearly that the misclassification rate achieved for all the mutual funds categories (except for the case of category I) is robust to the four different group of attributes used in this study.

Another very interesting issue is why these mutual funds (from category IX) are not classified in Group VII (FIM R.V. Euro), which has almost

\footnotetext{
${ }^{16}$ Authors are conscious that the FIM RV Insured, FIM RF Insured and Global Mutual Funds categories have different requisites from the rest of categories, and that may be the analysis of misclassified mutual funds should be done without considering them. So, we repeated all the analysis of step 1 without taking into account these categories. The results are very similar: In Group IX the percentage of funds correctly classified is $18 \%$, and the majority of them have a behavior like FIM RV International (Group X). However, the average misclassified mutual funds rates are almost identical (see Table 8(column two)). All results are available upon request.
}

identical requirements to the first one. The answer can be two-fold: First, it may be because Group VII does not have a sufficient amount of funds to make a group by themselves and to attract the mutual funds from Group IX. Secondly, it may be happening because the managers from Group IX are doing gaming activities so that they are changing their stated objectives towards more international objectives of investment.

In general we find that the average rate of mutual funds misclassified is about $40 \%$ (see Table 8 ), which is in accordance with related studies from the USA (e.g. Di Bartolomeo and Witkowski, 1997; or Kim et al., 2000). From this first analysis we could conclude that the stated objectives seem not to match with the ones the funds actually pursue. Thus, as mentioned previously, this could have a negative effect on the performance of a portfolio diversified between mutual funds categories, because an investor could try to diversify between official categories while they are not really different. In addition, it suggests that it might be needed to reduce the number of categories in the Spanish mutual funds classification. Thus, in the next step, we carry out another classification following a natural clustering technique, trying to answer these questions. 
Table 4

Percentage of mutual funds correctly classified according to KNN technique

\begin{tabular}{|c|c|c|c|c|c|c|c|c|c|c|c|c|c|c|}
\hline & I & II & III & IV & V & VI & VII & VIII & IX & $X$ & XI & XII & XIII & XIV \\
\hline I & 49.19 & 0.00 & 19.35 & 16.13 & 0.81 & 0.00 & 0.00 & 11.29 & 0.00 & 0.00 & 0.00 & 0.00 & 0.00 & 3.23 \\
\hline II & 1.75 & 7.02 & 7.02 & 5.26 & 5.26 & 0.00 & 3.51 & 17.54 & 0.00 & 21.05 & 8.77 & 15.79 & 5.26 & 1.75 \\
\hline IV & 8.00 & 0.00 & 6.00 & 54.00 & 2.00 & 2.00 & 0.00 & 14.00 & 0.00 & 0.00 & 0.00 & 0.00 & 0.00 & 14.00 \\
\hline V & 2.48 & 0.83 & 4.13 & 4.96 & 58.68 & 9.92 & 0.00 & 6.61 & 0.83 & 0.00 & 4.96 & 2.48 & 1.65 & 2.48 \\
\hline VI & 0.00 & 0.00 & 3.77 & 3.77 & 33.96 & 18.87 & 5.66 & 22.64 & 0.00 & 0.00 & 1.89 & 0.00 & 3.77 & 5.66 \\
\hline IX & 5.56 & 0.00 & 5.56 & 8.33 & 5.56 & 0.00 & 0.00 & 33.33 & 0.00 & 38.89 & 2.78 & 0.00 & 0.00 & 0.00 \\
\hline$X$ & 0.00 & 2.45 & 0.00 & 0.49 & 0.00 & 0.00 & 5.39 & 0.49 & 1.96 & 75.98 & 9.31 & 3.43 & 0.49 & 0.00 \\
\hline XI & 0.00 & 4.00 & 0.00 & 1.00 & 9.00 & 2.00 & 2.00 & 3.00 & 0.00 & 16.00 & 41.00 & 18.00 & 4.00 & 0.00 \\
\hline XII & 0.00 & 3.95 & 0.00 & 0.00 & 15.79 & 1.32 & 0.00 & 1.32 & 0.00 & 14.47 & 26.32 & 28.95 & 7.89 & 0.00 \\
\hline XIII & 0.00 & 4.17 & 0.00 & 4.17 & 18.75 & 0.00 & 0.00 & 4.17 & 0.00 & 4.17 & 8.33 & 20.83 & 20.83 & 14.58 \\
\hline
\end{tabular}

Employing five neighbours and the attributes of Group II.

This table shows the correspondence between the official categories and the attributed based classification. Each cell $(i, j)$ shows the percentage of the mutual funds from the legal category $i$ (rows) that are classified (from a $k$ nearest neighbours technique) in the category $j$ (columns). Thus, the diagonal (in boldface) represents the percentage of mutual funds which are correctly classified in the official classification.

Table 5

Percentage of mutual funds correctly classified according to KNN technique

\begin{tabular}{|c|c|c|c|c|c|c|c|c|c|c|c|c|c|c|}
\hline & I & II & III & IV & V & VI & VII & VIII & IX & X & XI & XII & XIII & XIV \\
\hline I & 79.84 & 0.00 & 12.90 & 5.65 & 0.00 & 0.00 & 0.00 & 0.81 & 0.00 & 0.00 & 0.00 & 0.00 & 0.00 & 0.81 \\
\hline II & 5.26 & 8.77 & 3.51 & 1.75 & 7.02 & 5.26 & 3.51 & 19.30 & 1.75 & 21.05 & 7.02 & 12.28 & 1.75 & 1.75 \\
\hline IV & 3.33 & 0.00 & 10.67 & 64.67 & 2.00 & 0.00 & 0.00 & 8.00 & 0.00 & 0.00 & 0.00 & 0.00 & 0.00 & 11.33 \\
\hline V & 0.83 & 0.83 & 1.65 & 4.13 & 61.98 & 9.92 & 0.83 & 8.26 & 0.83 & 0.00 & 5.79 & 1.65 & 2.48 & 0.83 \\
\hline VI & 0.00 & 0.00 & 1.89 & 0.00 & 56.60 & 9.43 & 3.77 & 16.98 & 0.00 & 0.00 & 0.00 & 1.89 & 7.55 & 1.89 \\
\hline IX & 0.00 & 0.00 & 0.00 & 0.00 & 0.00 & 2.78 & 0.00 & 55.56 & 0.00 & 36.11 & 5.56 & 0.00 & 0.00 & 0.00 \\
\hline $\mathrm{X}$ & 0.00 & 2.45 & 0.00 & 0.00 & 0.00 & 0.00 & 6.37 & 1.96 & 2.94 & 75.98 & 7.84 & 1.96 & 0.49 & 0.00 \\
\hline XI & 0.00 & 3.00 & 0.00 & 0.00 & 9.00 & 1.00 & 0.00 & 4.00 & 0.00 & 17.00 & 47.00 & 17.00 & 2.00 & 0.00 \\
\hline XII & 0.00 & 5.26 & 0.00 & 0.00 & 6.58 & 2.63 & 1.32 & 2.63 & 1.32 & 15.79 & 27.63 & 27.63 & 9.21 & 0.00 \\
\hline XIII & 0.00 & 2.08 & 4.17 & 0.00 & 4.17 & 4.17 & 4.17 & 6.25 & 0.00 & 2.08 & 4.17 & 20.83 & 31.25 & 16.67 \\
\hline
\end{tabular}

Employing five neighbours and the attributes of Group III.

This table shows the correspondence between the official categories and the attributed based classification. Each cell $(i, j)$ shows the percentage of the mutual funds from the legal category $i$ (rows) that are classified (from a $k$ nearest neighbours technique) in the category $j$ (columns). Thus, the diagonal (in boldface) represents the percentage of mutual funds which are correctly classified in the official classification.

\subsection{Building some new categories (Step 2)}

In this second step, we applied the above mentioned two-level approach, which is based on both the SOMs and the $k$-means clustering algorithm, trying to find a natural classification of Spanish mutual funds according to our four groups of attributes. We compute SOMs of different sizes 
Table 6

Percentage of mutual funds correctly classified according to KNN technique

\begin{tabular}{|c|c|c|c|c|c|c|c|c|c|c|c|c|c|c|}
\hline & I & II & III & IV & V & VI & VII & VIII & IX & $X$ & XI & XII & XIII & XIV \\
\hline I & 81.45 & 0.00 & 16.94 & 0.00 & 0.00 & 0.00 & 0.00 & 0.00 & 0.00 & 0.00 & 0.00 & 0.00 & 0.00 & 1.61 \\
\hline II & 5.26 & 12.28 & 3.51 & 0.00 & 7.02 & 0.00 & 3.51 & 24.56 & 0.00 & 26.32 & 7.02 & 7.02 & 3.51 & 0.00 \\
\hline III & 16.28 & 0.00 & 53.49 & 15.50 & 1.55 & 0.00 & 0.00 & 1.55 & 0.78 & 0.78 & 0.78 & 0.00 & 0.00 & 9.30 \\
\hline IV & 2.67 & 0.00 & 17.33 & 54.67 & 0.67 & 0.00 & 0.00 & 7.33 & 0.00 & 0.00 & 0.00 & 0.00 & 0.00 & 17.33 \\
\hline V & 0.00 & 1.65 & 4.13 & 4.96 & 57.85 & 11.57 & 1.65 & 5.79 & 0.83 & 0.00 & 5.79 & 1.65 & 3.31 & 0.83 \\
\hline VI & 0.00 & 1.89 & 0.00 & 0.00 & 52.83 & 11.32 & 1.89 & 15.09 & 3.77 & 0.00 & 1.89 & 0.00 & 11.32 & 0.00 \\
\hline VII & 0.00 & 0.00 & 0.00 & 1.41 & 5.63 & 2.82 & 52.11 & 4.23 & 0.00 & 22.54 & 4.23 & 5.63 & 1.41 & 0.00 \\
\hline VIII & 0.00 & 0.36 & 1.46 & 0.73 & 2.92 & 0.73 & 2.19 & 85.40 & 0.00 & 2.55 & 0.36 & 1.09 & 1.09 & 1.09 \\
\hline IX & 0.00 & 0.00 & 0.00 & 0.00 & 2.78 & 0.00 & 0.00 & 52.78 & 2.78 & 36.11 & 2.78 & 0.00 & 0.00 & 2.78 \\
\hline$X$ & 0.00 & 2.94 & 0.49 & 0.00 & 0.00 & 0.49 & 4.90 & 0.49 & 2.45 & 79.41 & 3.92 & 3.92 & 0.98 & 0.00 \\
\hline XI & 0.00 & 2.00 & 0.00 & 0.00 & 9.00 & 2.00 & 2.00 & 4.00 & 0.00 & 17.00 & 44.00 & 15.00 & 5.00 & 0.00 \\
\hline XII & 0.00 & 3.95 & 0.00 & 0.00 & 9.21 & 0.00 & 0.00 & 1.32 & 0.00 & 11.84 & 26.32 & 35.53 & 11.84 & 0.00 \\
\hline XIII & 0.00 & 4.17 & 0.00 & 0.00 & 16.67 & 4.17 & 0.00 & 2.08 & 2.08 & 8.33 & 12.50 & 16.67 & 18.75 & 14.58 \\
\hline XIV & 0.00 & 0.67 & 8.72 & 19.46 & 0.67 & 1.34 & 0.00 & 4.03 & 0.00 & 0.00 & 0.00 & 0.00 & 1.34 & 63.76 \\
\hline
\end{tabular}

Employing five neighbours and the attributes of Group IV.

This table shows the correspondence between the official categories and the attributed based classification. Each cell $(i, j)$ shows the percentage of the mutual funds from the legal category $i$ (rows) that are classified (from a $k$ nearest neighbours technique) in the category $j$ (columns). Thus, the diagonal (in boldface) represents the percentage of mutual funds which are correctly classified in the official classification.

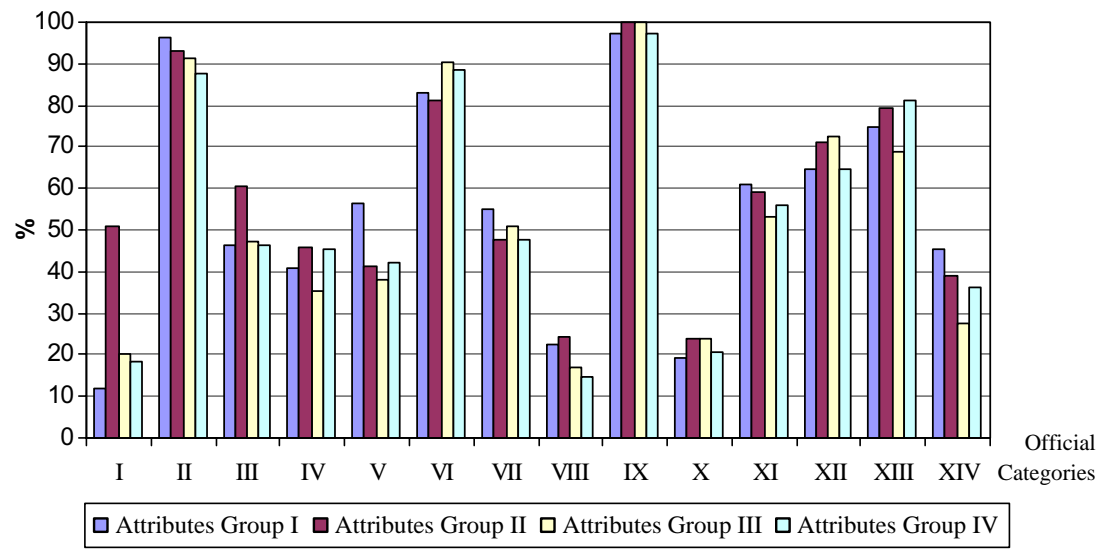

Fig. 1. Percentage of Mutual Funds Misclassified according to the KNN algorithm. This figure shows the rate of misclassified mutual funds according to the KNN algorithm. For each category of mutual funds (from I to XIV) we show the rate of misclassified funds using the four different groups of attributes (described in detail in Section 4.1).

$(5 \times 5,10 \times 10,10 \times 15,15 \times 15$ and $20 \times 20$ neurons) for each of the four groups of attributes. ${ }^{17}$

\footnotetext{
${ }^{17}$ The software used in this paper is the SOM Toolbox for Matlab from the Helsinki University of Technology (http:// www.cis.hut.fi/projects/somtoolbox/). All SOMs are linearly initialized and trained employing the batch algorithm. The training was performed in two phases: a rough training (in this phase we employ 500 epochs) and a fine tuning phase (here, we use 10.000 epochs).
}

From all experiments, we take the SOM configurations that have a higher number of clusters or, alternatively, the number of clusters which is more equally distributed (that is, which does not have a significant number of funds in a single cluster). Therefore, we choose as the best SOM clustering configuration: (i) a $10 \times 10 \mathrm{SOM}$ for the Group I of attributes; (ii) a $10 \times 15$ configuration for the Group II; (iii) a $15 \times 15$ for the Group III; and 
Table 7.1

Official categories classified in clusters according to a two level approach (SOMs and $k$ means) and with Group I of attributes

\begin{tabular}{|c|c|c|c|c|c|c|c|c|c|c|c|c|c|c|}
\hline Clusters & I & II & III & IV & V & VI & VII & VIII & IX & $X$ & XI & XII & XIII & XIV \\
\hline 1 & 0.00 & 5.26 & 0.00 & 1.33 & 40.50 & 47.17 & 11.27 & 20.44 & 13.89 & 1.47 & 3.00 & 7.89 & 37.50 & 6.71 \\
\hline 2 & 0.00 & 22.81 & 0.00 & 0.00 & 1.65 & 0.00 & 15.49 & 1.46 & 13.89 & 24.02 & 9.00 & 5.26 & 0.00 & 1.34 \\
\hline 3 & 0.00 & 42.11 & 2.33 & 5.33 & 20.66 & 16.98 & 64.79 & 6.57 & 25.00 & 73.53 & 87.00 & 86.84 & 50.00 & 8.05 \\
\hline 4 & 100.00 & 29.82 & 97.67 & 93.33 & 37.19 & 35.85 & 8.45 & 71.53 & 47.22 & 0.98 & 1.00 & 0.00 & 12.50 & 83.89 \\
\hline
\end{tabular}

This table shows the percentage of each official category (columns) that is place in each cluster (rows). These clusters are performed following a two level approach based on Self Organizing Maps (with a $10 \times 10$ neurons grid) and $k$ means clustering.

Table 7.2

Official categories classified in cluster according to a two level approach (SOMs and $k$ means) and with Group II of attributes

\begin{tabular}{|c|c|c|c|c|c|c|c|c|c|c|c|c|c|c|}
\hline Clusters & I & II & III & IV & V & VI & VII & VIII & IX & $\mathrm{X}$ & XI & XII & XIII & XIV \\
\hline 1 & 2.42 & 14.04 & 2.33 & 3.33 & 2.48 & 1.89 & 2.82 & 2.55 & 5.56 & 13.73 & 21.00 & 10.53 & 18.75 & 0.00 \\
\hline 2 & 16.94 & 10.53 & 24.81 & 24.67 & 6.61 & 7.55 & 2.82 & 40.15 & 22.22 & 0.98 & 2.00 & 0.00 & 2.08 & 10.07 \\
\hline 3 & 30.65 & 3.51 & 35.66 & 40.67 & 22.31 & 13.21 & 2.82 & 16.06 & 2.78 & 0.00 & 0.00 & 0.00 & 10.42 & 53.69 \\
\hline 4 & 11.29 & 31.58 & 9.30 & 3.33 & 47.93 & 54.72 & 18.31 & 11.68 & 13.89 & 20.59 & 48.00 & 63.16 & 54.17 & 3.36 \\
\hline 5 & 32.26 & 3.51 & 20.93 & 22.00 & 2.48 & 3.77 & 0.00 & 9.12 & 2.78 & 0.00 & 0.00 & 0.00 & 0.00 & 22.15 \\
\hline 6 & 0.00 & 33.33 & 2.33 & 2.00 & 4.96 & 7.55 & 54.93 & 14.96 & 44.44 & 47.55 & 25.00 & 23.68 & 6.25 & 0.67 \\
\hline 7 & 4.84 & 1.75 & 3.88 & 3.33 & 10.74 & 11.32 & 16.90 & 4.01 & 5.56 & 17.16 & 3.00 & 0.00 & 4.17 & 7.38 \\
\hline 8 & 1.61 & 1.75 & 0.78 & 0.67 & 2.48 & 0.00 & 1.41 & 1.46 & 2.78 & 0.00 & 1.00 & 2.63 & 4.17 & 2.68 \\
\hline
\end{tabular}

This table shows the percentage of each official category (columns) that is place in each cluster (rows). These clusters are performed following a two level approach based on Self Organizing Maps (with a $10 \times 15$ neurons grid) and $k$ means clustering.

(iv) $10 \times 10$ neurons for Group IV. From here and for the rest of the paper, to simplify we only employ these SOMs' configurations.

Finally, as the determination of the different clusters from the Kohonen Map is a complex and subjective task, following Vesanto and Alhoniemi (2000), we propose the following methodology. We take the SOM's output and configure the clusters employing the $k$-means algorithm. As the major drawback of $k$-means is that the number of clusters must be introduced by the researcher, we compute the $k$-means from 20 to 2 clusters, and calculate the Davies and Bouldin (1979) index. From this set, we choose that number of clusters which minimize the Davies Bouldin index. ${ }^{18}$

The results are shown in Tables 7.1 7.4. As we can see in Tables 7.1 7.4, in general, the natural number of clusters for the Spanish mutual funds industry is smaller than the 14 categories estab-

\footnotetext{
${ }^{18}$ As the $k$ means initialization is random, we repeat the above process 50 times for each SOM, to be sure that the number of clusters selected is the best one.
}

lished by the CNMV (or 11 if we do not consider the Global Mutual Funds, FIM RV Insured and FIM RF Insured). The number seems to be between 4 and 8 , but not 14 . Furthermore, from these tables, we can see how, generally, those categories that achieved the highest number of mutual funds correctly classified using the procedure proposed in the first step (categories I and X) also manage to put the highest proportion of mutual funds in a unique cluster in this second step, except when considering the second group of attributes. This could mean that these categories are compact and homogeneous, and should be maintained if an alternative classification is made. This new classification could be considered as an alternative for investors interested in the past performance of mutual funds and not on their portfolio composition, as it is the usual case for institutional investors such as Funds-of-Funds managers.

\subsection{Validation of the new classification (Step 3)}

After we have created a new mutual funds classification (for each one of the groups of attributes), 
Table 7.3

Official categories classified in cluster according to a two level approach (SOMs and $k$ means) and with Group III of attributes

\begin{tabular}{|c|c|c|c|c|c|c|c|c|c|c|c|c|c|c|}
\hline Clusters & I & II & III & IV & V & VI & VII & VIII & IX & $X$ & XI & XII & XIII & XIV \\
\hline 1 & 6.45 & 17.54 & 7.75 & 7.33 & 22.31 & 15.09 & 7.04 & 31.02 & 16.67 & 1.47 & 13.00 & 15.79 & 22.92 & 6.04 \\
\hline 2 & 62.90 & 10.53 & 40.31 & 70.00 & 25.62 & 18.87 & 5.63 & 2.19 & 2.78 & 1.96 & 25.00 & 22.37 & 4.17 & 73.15 \\
\hline 3 & 0.00 & 29.82 & 2.33 & 0.67 & 4.96 & 11.32 & 70.42 & 16.06 & 41.67 & 89.22 & 30.00 & 18.42 & 6.25 & 0.00 \\
\hline 4 & 0.81 & 14.04 & 10.08 & 2.67 & 5.79 & 5.66 & 5.63 & 10.22 & 13.89 & 2.94 & 3.00 & 0.00 & 6.25 & 0.67 \\
\hline 5 & 1.61 & 19.30 & 13.18 & 1.33 & 3.31 & 22.64 & 4.23 & 20.80 & 8.33 & 0.98 & 25.00 & 39.47 & 41.67 & 2.01 \\
\hline 6 & 20.16 & 3.51 & 10.85 & 4.00 & 0.00 & 1.89 & 1.41 & 1.82 & 2.78 & 0.00 & 0.00 & 0.00 & 0.00 & 1.34 \\
\hline 7 & 8.06 & 5.26 & 15.50 & 14.00 & 4.13 & 5.66 & 2.82 & 8.76 & 5.56 & 2.45 & 1.00 & 3.95 & 16.67 & 16.78 \\
\hline 8 & 0.00 & 0.00 & 0.00 & 0.00 & 33.88 & 18.87 & 2.82 & 9.12 & 8.33 & 0.98 & 3.00 & 0.00 & 2.08 & 0.00 \\
\hline
\end{tabular}

This table shows the percentage of each official category (columns) that is place in each cluster (rows). These clusters are performed following a two level approach based on Self Organizing Maps (with a $15 \times 15$ neurons grid) and $k$ means clustering.

Table 7.4

Official categories classified in cluster according to a two level approach (SOMs and $k$ means) and with Group IV of attributes

\begin{tabular}{|c|c|c|c|c|c|c|c|c|c|c|c|c|c|c|}
\hline Clusters & I & II & III & IV & V & VI & VII & VIII & IX & $X$ & XI & XII & XIII & XIV \\
\hline 1 & 97.58 & 7.02 & 75.97 & 48.67 & 30.58 & 28.30 & 1.41 & 25.91 & 25.00 & 0.00 & 1.00 & 0.00 & 12.50 & 75.17 \\
\hline 2 & 0.00 & 47.37 & 2.33 & 1.33 & 0.83 & 5.66 & 76.06 & 30.66 & 55.56 & 94.12 & 46.00 & 48.68 & 25.00 & 8.05 \\
\hline 3 & 2.42 & 14.04 & 12.40 & 32.00 & 22.31 & 20.75 & 7.04 & 18.98 & 11.11 & 1.96 & 44.00 & 36.84 & 22.92 & 14.77 \\
\hline 4 & 0.00 & 24.56 & 0.00 & 0.67 & 2.48 & 5.66 & 4.23 & 11.31 & 0.00 & 3.43 & 3.00 & 9.21 & 12.50 & 0.00 \\
\hline 5 & 0.00 & 7.02 & 9.30 & 17.33 & 43.80 & 39.62 & 11.27 & 13.14 & 8.33 & 0.49 & 6.00 & 5.26 & 27.08 & 2.01 \\
\hline
\end{tabular}

This table shows the percentage of each official category (columns) that is place in each cluster (rows). These clusters are performed following a two level approach based on Self Organizing Maps (with a $10 \times 10$ neurons grid) and $k$ means clustering.

Table 8

Average Percentage of Mutual Funds correctly classified from a methodology based on jacknife and KNN algorithm

\begin{tabular}{llll}
\hline & $\begin{array}{l}\text { Legal classification } \\
(\text { CNMV) }(\%)\end{array}$ & $\begin{array}{l}\text { Legal classification } \\
\text { (without categories II, IV and VIII) (\%) }\end{array}$ & $\begin{array}{l}\text { Classification from 2 level } \\
\text { approach (\%) }\end{array}$ \\
\hline Group I & 56.60 & 59.14 & 93.53 \\
Group II & 52.59 & 54.01 & 78.02 \\
Group III & 60.18 & 61.75 & 79.84 \\
Group IV & 59.30 & 61.03 & 85.30 \\
\hline
\end{tabular}

This table shows the average percentage of mutual funds correctly classified according to different groups of financial attributes (in rows). The first column represents the official classification in the Spanish market, the second one shows the results for the official classification without taking into account the categories II (Global Mutual Funds), IV (FIM RF Insured) and VIII (FIM RV Insured), the third one is the classification achieved from a methodology based on Self Organizing Maps and $k$ means clustering algorithm. This table shows the results obtained using a KNN algorithm with five neighbours.

we apply the same process as in step one to compute the number of funds misclassified from this natural classification with the object of analyzing if our new classification outperforms the official one. The results from this analysis are summarized in Table $8 .^{19}$

\footnotetext{
${ }^{19}$ To save space we only show the general results but more detailed results are available upon request.
}

As a general result, we can affirm that the average rate of misclassifications is lower than from the 14 official categories (Tables 3 6). Now, the average rate of mutual funds correctly classified is $93.53 \%, 78.02 \%, 79.84 \%$ and $85.30 \%$, considering each one of the group of attributes (see Table 8 (column 3)). Moreover, it seems that the second group of attributes (which adds the skewness and kurtosis over the first group of attributes) does 


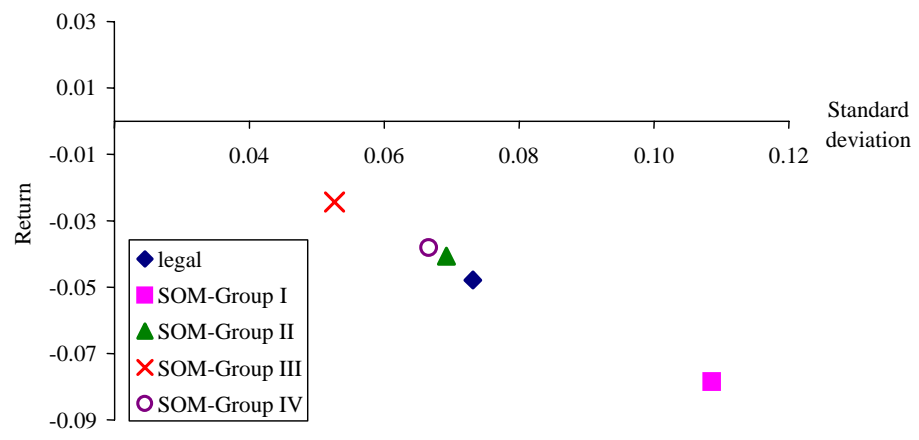

Fig. 2. Risk/return results from simulations of random equally weighted portfolios. This figure shows the average results obtained from the simulations of equally weighted portfolios. In each simulation, the random portfolios are composed of a mutual fund from each one of the categories (the official classification or those classifications obtained from the two level technique and each one of the group of financial attributes).

not do a good job of classifying mutual funds, even though it outperforms the official classification. It could mean that kurtosis and skewness do not represent attributes which allow to distinguish between different styles of investment in the mutual fund industry.

Although the above analysis seems to be very relevant from an academic perspective, investors could not be very interested in which is the misclassification rate of mutual funds according to a statistical criterion. They could be much more interested in which classification is better from a financial perspective, that is, which classification is better to achieve an optimal diversified portfolio. For this reason we perform the following analysis.

\subsection{An analysis of the diversification effect (Step 4)}

Now, we adopt the investor's perspective and we suppose that we decide to make an equally weighted portfolio from each of the categories of mutual funds. ${ }^{20}$ The procedure is as follows: We chose randomly a mutual fund from each of the 14 official categories. Next, we do the same for the clusters obtained from the two-level approach (step 2). Note that here we chose only a mutual fund from each cluster, so the number of assets

\footnotetext{
${ }^{20}$ Experiments like that were done by some other authors: Gallo and Lockwood (1997) or Di Bartolomeo and Witkowski (1997).
}

in these portfolios is lower than in the official classification. This random process is repeated 10.000 times and the portfolios' performances are evaluated under a mean-variance framework.

In Fig. 2 we show the average results from these equally weighted portfolios. As can be seen, in general, the new classification from SOM and the $k$-mean algorithm (even the number of asset in each portfolio is lower) achieves better results in terms of return-risk. ${ }^{21}$ Only in the case where we classify using the first group of attributes (risk and returns measures) the portfolio obtained from the official classification is not worse. However, we must realize that in the case of the Group I the total number of clusters is rather low, it has only four clusters, and therefore, the portfolio is comprised of only four assets while the portfolio from the official classification has 14 assets.

Summarizing, we can conclude that the Spanish official classification which is based on the mutual funds stated objectives when they were created, seems to be misleading. It may have an excessive number of categories, the effect of which is a reduction in the diversification effect that an investor can achieve using it.

\footnotetext{
${ }^{21}$ It must be noted that although the average results are all negative, it is normal given that we are using a sample where the market was bearish and so the majority of mutual funds achieved negative returns.
} 


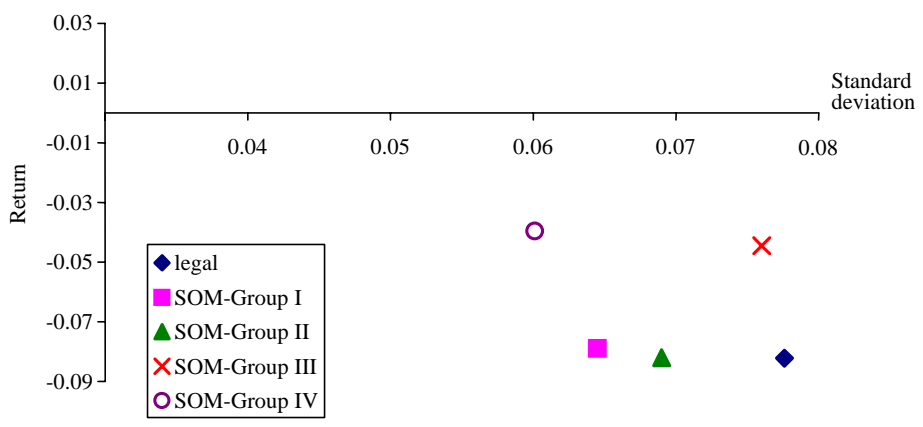

Fig. 3. Risk/return results from simulations of random equally weighted portfolios for an ex ante analysis. This figure shows the average results obtained from the simulations of equally weighted portfolios. In each simulation, the random portfolios are composed of a mutual fund from each one of the categories (the official classification or those classifications obtained from the two level technique and each one of the group of financial attributes). The classification from the two level technique is obtained from a first sub sample (January 2000 December 2001) and the portfolios are evaluated in the next sub sample (2002).

\subsection{An economical validation of the new classification (Step 5)}

In this last part of the paper, we present an alternative and complementary analysis of this new methodology to classify mutual funds. Given that the analysis realized in the previous Section 4.4 is based on an ex post analysis, the results from the new classification presented here could be biased in favor of performing better than the legal classification. Thus, in this section we repeat the previous analysis but dividing the sample in two different sub-samples. The first sub-sample is used as a pre-period for classifying the mutual funds and it includes data from January 2000 until December 2001. The second sub-sample (from January 2002 to January 2003) is used as a postperiod to evaluate the funds classifications obtained in the first sub-sample.

The procedure is as follows. First, for the preperiod we repeat step 2 and obtain the new classification using the two-level method, based in the SOM and the $k$-means algorithm. Second, we compute equally weighted portfolio from each one of the categories of mutual funds for both the new classification based on the two-level model and the official classification. As in the previous section we choose the funds in each portfolio randomly and we repeat it 10,000 times. But now we compute their average performances for the next time period (the second sub-sample), thus, we adopt the perspective of an investor who wishes to invest in mutual funds and does not know their performance in the next year. The results are shown in Fig. 3.

As we can observe in Fig. 3, independently of the group of funds attributes considered, the new classification of mutual funds outperforms the official ones in terms of risk and return. We can see again how the classifications obtained from the Group of attributes III and IV achieve the best performance. In these cases we reduce the losses from $8 \%$ to $4 \%$ and we also reduce the standard deviation from almost $8 \%$ to $6 \%$. In no case the new classification obtained using SOM is worse than the official one.

\section{Conclusions}

In this paper, we analyze whether Spanish mutual funds are misclassified according to the actual official classification from CNMV, which is based on the investment objectives originally declared by the fund. We find that only an average of $60 \%$ of the mutual funds is correctly classified according to their financial attributes (risk/return, correlations between categories and several performance measures). We also find that this erroneous classification may be due to an excess of 
categories. Some of the categories based on the investment objectives are not sufficiently different from others in financial terms; therefore, they may reduce the diversification effect. A correct mutual funds' classification is crucial for different reasons: making benchmarks correctly, studying mutual fund persistence, measuring performances from mutual funds, as well as for every topic related with performance measures.

We investigate whether it is possible to establish a new classification according to the mutual fund attributes, which has a lower rate of misclassified funds. To do this, we employ a two-level approach, which is based on Self-Organizing Maps (a type of unsupervised Artificial Neural Nets) and $k$-means (a popular clustering algorithm). We find that, for the period considered, it attains a lower rate of misclassified funds. Also, we find that the number of optimal categories must be between four and nine, but never fourteen. Note however that these results should be interpreted with caution: alternative classification algorithms such as decision trees, C4.5, etc. as well as other datasets, periods of time, or frequencies might have been used.

From an investor's perspective, it is more relevant to have a classification which permits an efficient diversification and to achieve a good performance in terms of risk/return. We do an application to portfolio selection, simulating equally weighted portfolios where each portfolio is compounded by only one mutual fund from each category. We find that the new classification outperforms the official one, in terms of return and risk, for almost all specifications and for both an in-sample and an out-sample analysis.

\section{Acknowledgements}

We would like to thank participants in the seminar organized at the Universidad de Valencia (Spain, 2003), and in the XI Financial Forum in Alicante (Spain, 2003) and the 11th International Conference Forecasting Financial Markets (Paris, 2004) for their helpful comments. All errors are the sole responsibility of the authors. David Moreno acknowledges the financial support provided by Ministerio de Educación y Cultura grant SEJ2004-01688/ECON.

\section{References}

Aha, D.W., 1990. A Study of Instance Based Algorithms for Supervised Learning Tasks, Technical Report, University of California, Irvine.

Bezdek, J.C., Pal, N.R., 1998. Some new indexes of cluster validity. IEEE Transactions on Systems, Man and Cyber netics 28, 301315 .

Brown, S.J., Goetzmann, W.N., 1995. Performance persistence. Journal of Finance 50 (2), 679698.

Brown, S.J., Goetzmann, W.N., 1997. Mutual fund styles. Journal of Financial Economics 43 (3), 373399.

Brown, S.J., Goetzmann, W.N., Ibbotson, R.G., Ross, S.A., 1992. Survivorship bias in performance studies. Review of Financial Studies 5, 553580.

Carhart, M.M., 1997. On persistence in mutual fund perfor mance. Journal of Finance 52 (1), 5782.

Dasarathy, B.V., 1991. Nearest Neighbor (NN) Norms: NN Pattern Classification Techniques. IEEE Computer Society Press.

Davies, D.L., Bouldin, D.W., 1979. A cluster separation measure. IEEE Transactions on Pattern Analysis and Machine Intelligence 1, 224227.

Deboeck, G., Kohonen, T., 2000. Visual Explorations in Finance and Investments Using Self Organizing Maps. Springer Verlag, Berlin.

Di Bartolomeo, D., Witkowski, E., 1997. Mutual fund misclassification: Evidence based on style analysis. Financial Analysts Journal (Sep/Oct), 3243.

Efron, B., Tibshirani, R., 1993. An Introduction to the Bootstrap. Chapman \& Hall CRP, cop.

Gallo, J., Lockwood, L.J., 1997. Benefits of proper style classification of equity managers. Journal of Portfolio Management 23 (3), 4755.

Gray, R.M., 1984. Vector quantization. IEEE ASSP Magazine 1 (April), 429.

Grinblatt, M., Titman, S., 1992. The persistence of mutual fund performance. Journal of Finance 47, 19771984.

Grinblatt, M., Titman, S., 1994. A study of monthly mutual fund returns and performance evaluation techniques. Jour nal of Financial and Quantitative Analysis 29 (3), 419444.

Kaski, S., 1997. Data Exploration Using Self Organizing Maps. $\mathrm{Ph}$. Thesis, Helsinki University of Technology. Acta Poly technica Scandinavica: Mathematics, Computing and Man agement in Engineering.

Kim, M., Shukla, R., Thomas, M., 2000. Mutual fund objective misclassification. Journal of Economics and Business 52 (4), 309324.

Kohonen, T., 1997. Self organizing Maps. Springer, Berlín.

Marathe, A., Shawky, H.A., 1999. Categorizing mutual funds using clusters. Advances in Quantitative Analysis of Finance and Accounting 7 (1), 199204. 
Sharpe, W.F., 1964. Capital asset prices: A theory of market equilibrium under conditions of risk. Journal of Finance 19 (3), 425442.

Sharpe, W.F., 1992. Assets allocation: Management style and performance measurement. Journal of Portfolio Manage ment 18 (2), 719.
Tou, J.T., González, R.C., 1974. Pattern Recognition Princi ples. Addison Wesley, Massachusetts.

Vesanto, J., Alhoniemi, E., 2000. Clustering of the self organizing map. IEEE Transactions on Neural Networks 11 (3), 586600. 